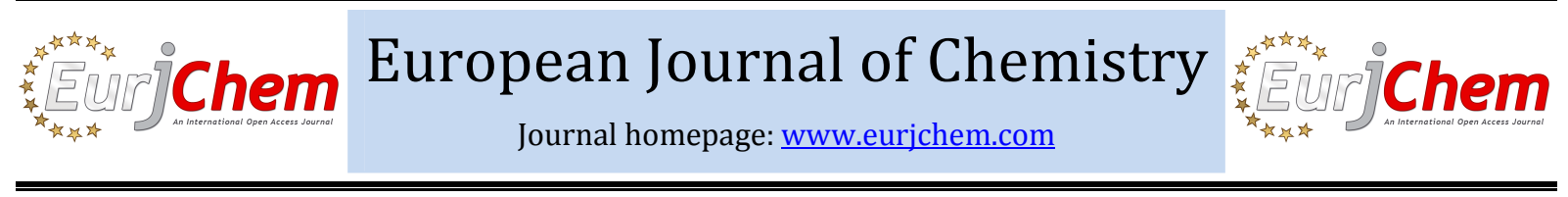

\title{
An environmentally benign and efficient synthesis of 2-thio-substituted benzothiazoles
}

\author{
Gaddam Satish, Kasireddy Harsha Vardhan Reddy, Katla Ramesh, \\ Jilla Shankar, and Yadavalli Venkata Durga Nageswar*
}

Council of Scientific and Industrial Research, Medicinal Chemistry and Pharmacology Division, Indian Institute of Chemical Technology, Uppal Road, Hyderabad 500-007, India

${ }^{*}$ Corresponding author at: Council of Scientific and Industrial Research, Medicinal Chemistry and Pharmacology Division, Indian Institute of Chemical Technology, Uppal Road, Hyderabad 500-007, India.

Tel.: +91.40.2791654.Fax: +91.40.27193189. E-mail address: dryvdnageswar@gmail.com (Y.V.D. Nageswar).

\section{ARTICLE INFORMATION}

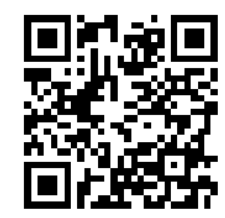

DOI: $10.5155 /$ eurjchem.5.2.291-295.861

Received: 24 June 2013

Received in revised form: 27 July 2013

Accepted: 31 July 2013

Online: 30 June 2014

\section{KEYWORDS}

\section{$\mathrm{CuFe}_{2} \mathrm{O}_{4}$}

PEG-400

Benzothiazole

Iodo benzenes

Magnetically separable

2-Mercapto benzothiazole

\section{Introduction}

In the area of heterocyclic chemistry, fused five member systems are important building blocks, and among these 2-thiosubstituted benzothiazoles have been designed and synthesized for biological evaluation. 2-Thio-substituted-1,3benzothiazoles are significant scaffolds found in a large number of pharmaceutically active molecules [1-6]. These include Cathepsin-D inhibitor, potent heat shock protein-90 inhibitor, avarol-3'-thiobenzothiazole, 2-(thiocyanatomethylthio)-1,3benzothiazole, and dual antagonist for the human CCR1 and CCR3 receptors [7,8]. 2-Thio-substituted-1,3-benzothiazoles have also been found in advanced materials used as corrosion inhibitors and vulcanization catalysts in the rubber industry and reagents for metal-catalyzed cross-coupling reactions. Some of the biologically active 2-thio-substituted benzothiazoles are given in Figure 1. Due to the significance attached to 2-thio-substituted benzothiazoles many research groups are attracted towards exploring various ecofriendly protocols [9]. Recently green chemistry has attracted the attention of all research groups to peruse organic synthetic transformations in sustainable reaction mediums [10].
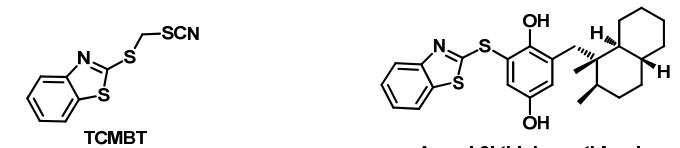

Avarol-3'-thiobenzothiazole

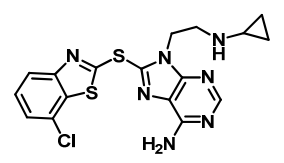

Heat Shock protein (HSP)-90-inhibitor<smiles>O=C(Nc1ccc(Sc2nc3ccc(OC(F)(F)F)cc3s2)cc1)c1cc(Cl)cc(Cl)c1O</smiles>

Cathepsin-D-Inhibitor
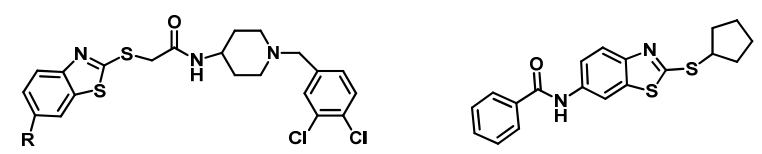

Human CCR1, CCR3 Receptor antagonist $\quad N-(2-$-(Cyclopentylthio)benzo[d]thiazol-6-yl)ber

Figure 1. Biologically active $N$-substituted-2-aminobenzothiazole derivatives. 
Polyethylene glycol (PEG) is a polymerized compound of ethylene oxide, which is hydrophilic in nature. It has benign characteristic properties with respect to environment and chemical industry such as low cost, low flammability, low toxicity, recyclability, facile degradability, and miscibility with various organic solvents likes toluene, dichloromethane, alcohol, and acetone [11], compared to ionic liquids, supercritical fluids, and micellar systems [12]. Polyethylene glycol is compatible with several organic synthetic transformations like substitution reactions [13], oxidation and reduction reactions [14], Heck reaction [15], asymmetric dihydroxylation [16], Suzuki cross-coupling reaction [17], Wacker reaction [18], and partial reductions of alkynes [19].

The fascinating area of cross-coupling chemistry led many research groups to develop new catalytic systems to obtain several libraries of new molecules. Due to various applications associated with 2-thio-substituted benzothiazoles, C-S bond formation through coupling reaction using copper has been developed [20]. Recently, Wei-Yi Wu and co-workers described the synthesis of 2-thiosubstituted benzothiazole by $\mathrm{FeCl}_{3} .6 \mathrm{H}_{2} \mathrm{O}$ catalyzed C-S bond formation from 2-mercapto benzothiazole with corresponding aryl halide [21]. Even though, the reported methods serve the purpose of obtaining 2-thio-substituted benzothiazoles, these reported catalytic systems are not recyclable.

In continuation of our work in the field of cross-coupling reactions [22-30] herein, we describe an inexpensive, air-stable and recyclable nanocopper ferrite as a catalyst for the synthesis of 2-thio-substituted benzothiazoles under ligand-free conditions. Heterogeneous catalysts are useful both from economic and industrial point of view when compared to homogeneous catalysts. The high surface area and reactive morphologies of nanomaterials allow them to be effective catalysts for organic synthesis. Copper ferrite nanoparticles $\left(\mathrm{CuFe}_{2} \mathrm{O}_{4}\right.$ nanoparticles) have the advantages of recyclability, easy workup, and cleaner reaction profiles apart from lack of nessesity of external ligands minimizing the organic waste generation as compared to the conventional catalytic systems. A mixture of 2-iodo aniline $(0.5 \mathrm{mmol})$, carbon disulfide $(0.6$ mmol), $\mathrm{CuFe}_{2} \mathrm{O}_{4}$ nanoparticles $(0.06 \mathrm{mmol})$, and $\mathrm{Cs}_{2} \mathrm{CO}_{3}(1.5$ mmol) in PEG-400 (3 mL) was stirred at $90{ }^{\circ} \mathrm{C}$ for $8 \mathrm{~h}$. The product 2-mercapto benzothiazole was synthesized in good to excellent yield. The results are included in Table 1 . The identity and purity of the product was confirmed by ${ }^{1} \mathrm{H}$ NMR, and ESIMS and compared with authentic samples in literature [31].

\section{Experimental}

All materials were purchased from Sigma Aldrich. Dry solvents, $\mathrm{CuFe}_{2} \mathrm{O}_{4}$ nanopowder $(<50 \mathrm{~nm})$ were used for the reactions. Column chromatography was carried out using silica gel (60-120 mesh size). Analytical thin layer chromatography (TLC) was carried out using silica gel $60 \mathrm{~F}_{254}$ pre-coated plates. Visualization was accomplished with UV lamp, I2 stain, and phosphomolybdic acid charring. All the products were characterized by their NMR (Varian 200 or Avance 300 spectrometer) and Mass spectra (VG Autospec). ${ }^{1} \mathrm{H}$ NMR and ${ }^{13} \mathrm{C} \mathrm{NMR}$ were recorded on 200 or $300 \mathrm{MHz}$ in $\mathrm{CDCl}_{3}$, and the chemical shifts were reported in parts per million (ppm, $\delta$ ) downfield from the tetramethylsilane. Scanning electron microscopy (SEM) analyses were performed using a Make: Hitachi S- 3000N scanning electron microscope. Powder X-ray diffraction (XRD) analyses were performed using a Make: Bruker, Model: D8- Advance, Detector: Lynx-Eye.

\subsection{Recovering of $\mathrm{CuFe}_{2} \mathrm{O}_{4}$ nanoparticles}

The reusability of the nano copper ferrite catalyst was examined, after completion of the reaction, $\mathrm{CuFe}_{2} \mathrm{O}_{4}$ nano particles were allowed to be on the bottom of the flask by a neodymium magnet, and the supernatant solution was removed. The separated nano catalyst was washed successively with $10 \mathrm{~mL}$ of water, ethanol, ethyl acetate, dichloromethane, and hexane and then dried. No significant loss of catalyst activity was observed up to four cycles. It was observed from the SEM studies (Figure 2) that the used $\mathrm{CuFe}_{2} \mathrm{O}_{4}$ nanoparticles were similar in morphology, to the native catalyst even after four cycles.

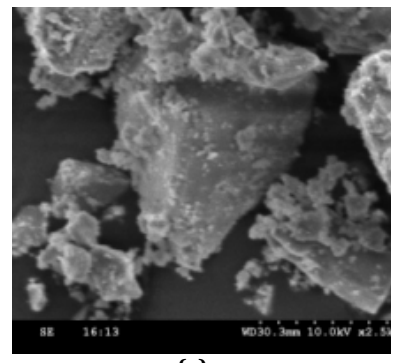

(a)

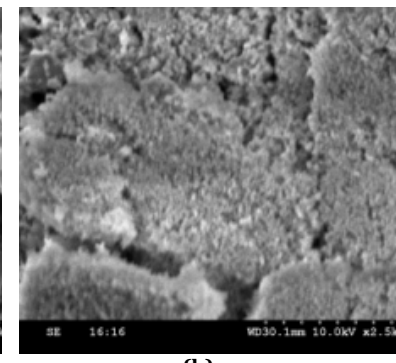

(b)
Figure 2. SEM images of $\mathrm{CuFe}_{2} \mathrm{O}_{4}$ nanoparticles (a) native $\mathrm{CuFe}_{2} \mathrm{O}_{3}$ nanoparticles, (b) $\mathrm{CuFe}_{2} \mathrm{O}_{4}$ nanoparticles after four cycles.

\subsection{General procedure for the synthesis of 2-thio-substituted benzothiazole derivatives}

A mixture of 2-mercapto benzothiazole $(0.5 \mathrm{mmol})$, iodo benzene $(0.6 \mathrm{mmol}), \mathrm{CuFe}_{2} \mathrm{O}_{4}$ nanoparticles $(0.06 \mathrm{mmol})$, and $\mathrm{Cs}_{2} \mathrm{CO}_{3}(1.3 \mathrm{mmol})$ in PEG-400 $(3 \mathrm{~mL})$ was stirred at $90{ }^{\circ} \mathrm{C}$ for 8 h. After completion of the reaction, $\mathrm{CuFe}_{2} \mathrm{O}_{4}$ nano particles were allowed to be at the bottom of the flask by a neodymium magnet, and the supernatant solution was removed. The supernatant solution was extracted with ethyl acetate and the organic layer was washed with water and brine, and then dried over $\mathrm{Na}_{2} \mathrm{SO}_{4}$. After removal of the solvent in vacuum, the residue was purified by silica-gel chromatography to give the desired 2-thio-substituted benzothiazoles. The identity and purity of the product was confirmed by ${ }^{1} \mathrm{H}$ NMR, ${ }^{13} \mathrm{C}$ NMR and ESI-MS.

\subsection{General procedure for the synthesis of 2-mercapto benzothiazole derivatives}

A mixture of 2-iodo aniline (0.5 mmol), carbon disulfide (0.6 mmol), $\mathrm{CuFe}_{2} \mathrm{O}_{4}$ nanoparticles $(0.06 \mathrm{mmol})$, and $\mathrm{Cs}_{2} \mathrm{CO}_{3}$ (1.5 mmol) in PEG-400 (3 mL) was stirred at $90^{\circ} \mathrm{C}$ for $5 \mathrm{~h}$. After the reaction, after completion of the reaction, $\mathrm{CuFe}_{2} \mathrm{O}_{4}$ nano particles were allowed to be at the bottom of the flask by a neodymium magnet, and the supernatant solution was removed. The supernatant solution was extracted with ethyl acetate and the organic layer was washed with water and brine, and then dried over $\mathrm{Na}_{2} \mathrm{SO}_{4}$. After removal of the solvent in vacuum, the residue was purified by silica-gel chromatography to give the desired substituted 2-mercapto benzothiazoles in good to excellent yields (Scheme 1).

2-(Phenylthio)benzo[d]thiazole (Table 1, Entry 1, 3a): ${ }^{1} \mathrm{H}$ NMR (300 MHz, $\left.\mathrm{CDCl}_{3}, \delta, \mathrm{ppm}\right) 7.89$ (d, 1H, $\left.J=8.3 \mathrm{~Hz}, \mathrm{Ar}-\mathrm{H}\right)$, 7.79-7.72 (m, 2H, Ar-H), 7.66 (d, 1H, J = 8.3 Hz, Ar-H), 7.54-7.46 (m, 3H, Ar-H), 7.45-7.38 (m, 1H, Ar-H) 7.32-7.27 (m, 1H, Ar-H). ${ }^{13} \mathrm{C}$ NMR $\left(75 \mathrm{MHz}, \mathrm{CDCl}_{3}, \delta, \mathrm{ppm}\right): 135.3,130.4,129.9,126.1$, 124.31, 121.9, 120.7 (Ar-C). ESI-MS (m/z): $244(\mathrm{M}+\mathrm{H})^{+}$.

2-(Phenylthio)-6-(trifluoromethyl)benzo[d] thiazole (Table 1, Entry 2, 3b): ${ }^{1} \mathrm{H}$ NMR $\left(300 \mathrm{MHz}, \mathrm{CDCl}_{3}, \delta, \mathrm{ppm}\right): 7.66$ (s, $1 \mathrm{H}, \mathrm{Ar}-$ H), 7.57-7.43 (d, 1H, Ar-H), 7.30-7.23 (m, 2H, Ar-H), 7.20-7.07 (m, 3H, Ar-H), $6.81(\mathrm{~d}, 1 \mathrm{H}, J=9.0 \mathrm{~Hz}, \mathrm{Ar}-\mathrm{H}) .{ }^{13} \mathrm{C}$ NMR $(75 \mathrm{MHz}$, $\left.\mathrm{CDCl}_{3}, \delta, \mathrm{ppm}\right): 114.5$ (Ar-C), 120.6 (Ar-C), $123.5\left(\mathrm{CF}_{3}-\mathrm{C}\right), 124.3$ (Ar-C), 125.9 (Ar-C), 126.7 (Ar-C), 129.1 (Ar-C), 134.5 (Ar-C), 140.4 (Ar-C), 153.9 (Ar-C). ESI-MS (m/z): $312(\mathrm{M}+\mathrm{H})^{+}$. 
Table 1. Synthesis of 2-phenyl-2,3-dihydroquinazolin-4(1H)-one derivatives a.

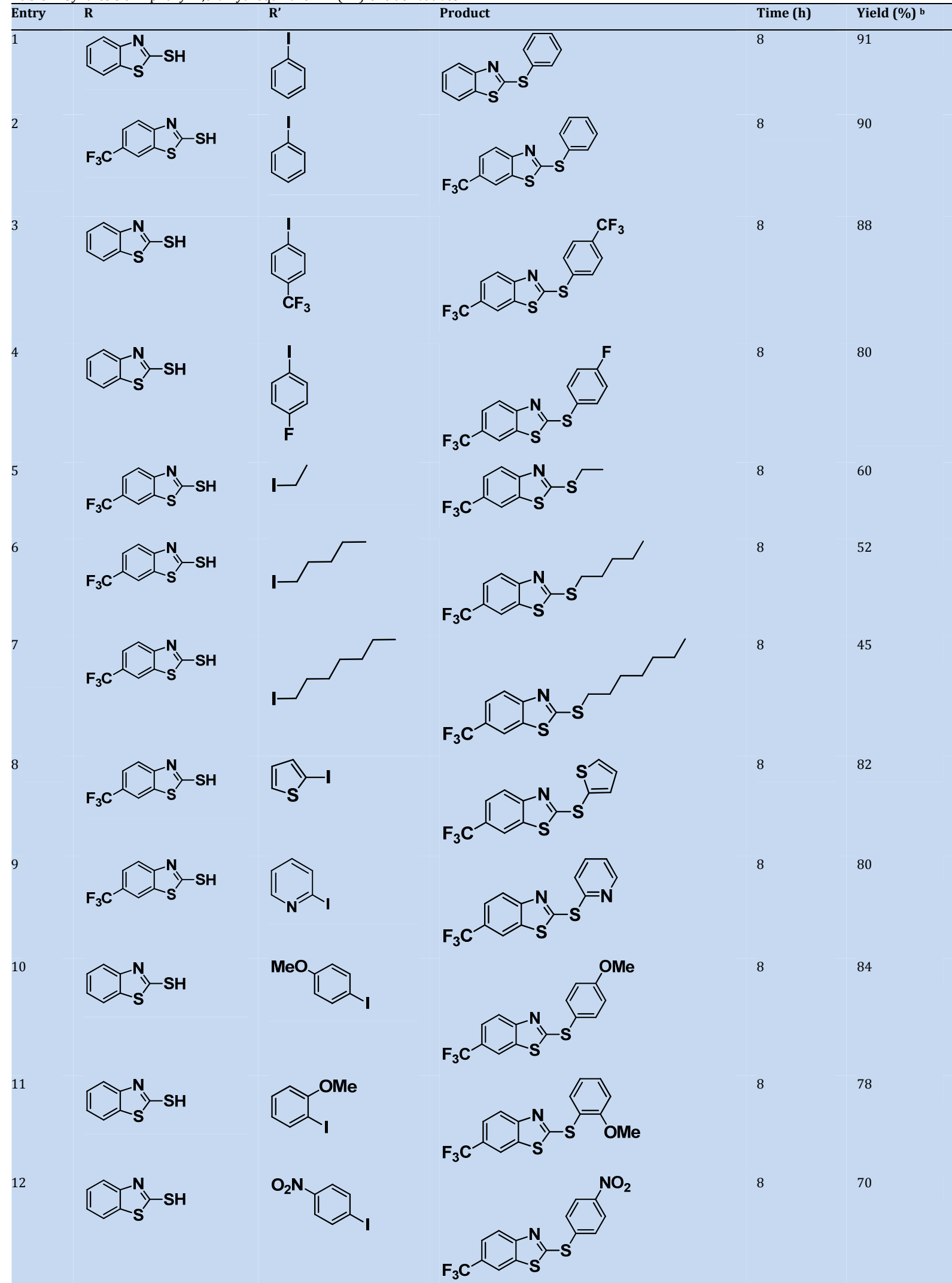

a Reaction conditions: A mixture of 2-mercapto benzothiazole ( $0.5 \mathrm{mmol})$, iodo benzene (0.6 mmol), $\mathrm{CuFe}_{2} \mathrm{O}_{4}$ nanoparticles $\left(0.06 \mathrm{mmol}\right.$, and $\mathrm{Cs}_{2} \mathrm{CO}_{3}(1.3 \mathrm{mmol})$ in PEG-400 (3 mL) was stirred at $90^{\circ} \mathrm{C}$ for $8 \mathrm{~h}$. 

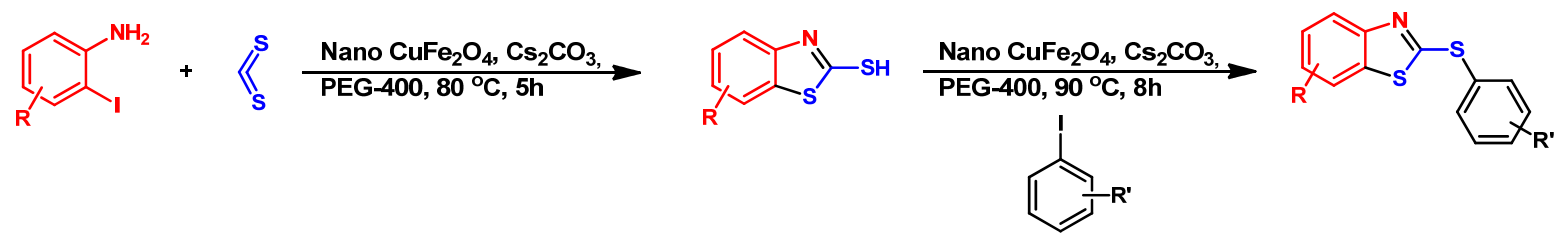

Scheme 1

Table 2. Screening of nanoparticles for the synthesis of 2-thio-substituted benzothiazoles a.

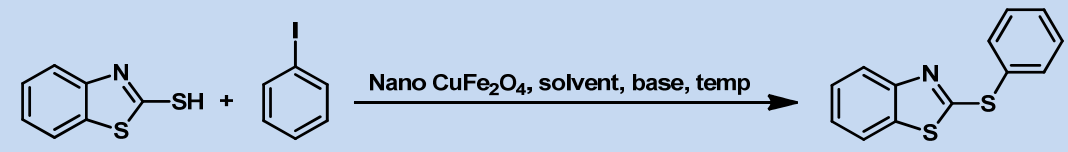

\begin{tabular}{|c|c|c|c|c|c|}
\hline Entry & Catalyst & Base & Solvent & Temperature $\left({ }^{\circ} \mathrm{C}\right)$ & Yield (\%) b \\
\hline 1 & Nano $\mathrm{CuFe}_{2} \mathrm{O}_{4}$ & $\mathrm{Cs}_{2} \mathrm{CO}_{3}$ & PEG & 90 & 91 \\
\hline 2 & Nano $\mathrm{CuFe}_{2} \mathrm{O}_{4}$ & $\mathrm{~K}_{2} \mathrm{CO}_{3}$ & PEG & 90 & 40 \\
\hline 3 & Nano $\mathrm{CuFe}_{2} \mathrm{O}_{4}$ & $\mathrm{Na}_{2} \mathrm{CO}_{3}$ & PEG & 90 & 20 \\
\hline 4 & Nano $\mathrm{CuFe}_{2} \mathrm{O}_{4}$ & $\mathrm{~K}_{3} \mathrm{PO}_{4}$ & PEG & 90 & 40 \\
\hline 5 & Nano $\mathrm{CuFe}_{2} \mathrm{O}_{4}$ & $\mathrm{Cs}_{2} \mathrm{CO}_{3}$ & PEG & Room temperature & Trace \\
\hline 6 & Nano $\mathrm{CuFe}_{2} \mathrm{O}_{4}$ & $\mathrm{Cs}_{2} \mathrm{CO}_{3}$ & $\mathrm{CH}_{3} \mathrm{CN}$ & 90 & 25 \\
\hline 7 & Nano $\mathrm{CuFe}_{2} \mathrm{O}_{4}$ & $\mathrm{Cs}_{2} \mathrm{CO}_{3}$ & Toluene & 90 & 30 \\
\hline 8 & Nano $\mathrm{CuFe}_{2} \mathrm{O}_{4}$ & $\mathrm{Cs}_{2} \mathrm{CO}_{3}$ & THF & 90 & 15 \\
\hline 9 & Nano $\mathrm{CuFe}_{2} \mathrm{O}_{4}$ & $\mathrm{Cs}_{2} \mathrm{CO}_{3}$ & PEG & 50 & 20 \\
\hline 10 & Nano $\mathrm{CuFe}_{2} \mathrm{O}_{4}$ & - & PEG & 90 & $0^{c}$ \\
\hline 11 & Nano $\mathrm{CuFe}_{2} \mathrm{O}_{4}$ & $\mathrm{Cs}_{2} \mathrm{CO}_{3}$ & $\mathrm{H}_{2} \mathrm{O}$ & 90 & 0 \\
\hline 12 & - & $\mathrm{Cs}_{2} \mathrm{CO}_{3}$ & PEG & 90 & $0 \mathrm{~d}$ \\
\hline 13 & Nano $\mathrm{Y}_{2} \mathrm{O}_{3}$ & $\mathrm{Cs}_{2} \mathrm{CO}_{3}$ & PEG & 50 & 15 \\
\hline 14 & Nano NiO & $\mathrm{Cs}_{2} \mathrm{CO}_{3}$ & PEG & 50 & 10 \\
\hline 15 & Nano $\mathrm{Co}_{3} \mathrm{O}_{4}$ & $\mathrm{Cs}_{2} \mathrm{CO}_{3}$ & PEG & 50 & Trace \\
\hline 16 & Nano $\mathrm{NiFe}_{2} \mathrm{O}_{4}$ & $\mathrm{Cs}_{2} \mathrm{CO}_{3}$ & PEG & 50 & 13 \\
\hline
\end{tabular}

in PEG-400 (3 mL) was stirred at $90^{\circ} \mathrm{C}$ for $8 \mathrm{~h}$.

b Isolated yield.

c In absence of the catalyst.

${ }^{d}$ In absence of the base.

2-((4-(Trifluoromethyl)phenyl)thio)benzo[d]thiazole (Table 1, Entry 3, 3c): ${ }^{1} \mathrm{H}$ NMR $\left(300 \mathrm{MHz}, \mathrm{CDCl}_{3}, \delta, \mathrm{ppm}\right): 7.91(\mathrm{~d}, 1 \mathrm{H}, J$ = 7.9 Hz, Ar-H), 7.90-7.79 (d, 2H, Ar-H), 7.78-7.66 (m, 2H, Ar-H), 7.55-7.42 (m, 2H, Ar-H) 7.41-7.32 (m, 1H, Ar-H). ESI-MS $(\mathrm{m} / \mathrm{z})$ : $312(\mathrm{M}+\mathrm{H})^{+}$.

2-((4-Fluorophenyl)thio)benzo[d]thiazole (Table 1, Entry 4, 3d): ${ }^{1} \mathrm{H}$ NMR (300 MHz, $\left.\mathrm{CDCl}_{3}, \delta, \mathrm{ppm}\right): 9.03$ (s, $\left.1 \mathrm{H}, \mathrm{Ar}-\mathrm{H}\right), 8.15$ (d, $2 \mathrm{H}, J=8.3 \mathrm{~Hz}, \mathrm{Ar}-\mathrm{H}$ ), 7.98 (d, $2 \mathrm{H}, J=7.5 \mathrm{~Hz}, \mathrm{Ar}-\mathrm{H}), 7.64-7.43$ (m, 3H, Ar-H). ${ }^{13} \mathrm{C}$ NMR (75 MHz, $\mathrm{CDCl}_{3}, \delta$, ppm): 163.8 (Ar-C), 151.3, F-C, 135.6 (Ar-C), 126.1 (Ar-C), 125.5 (Ar-C), 123.5 (ArC), $121.8\left(\right.$ Ar-C). ESI-MS $(m / z): 262(\mathrm{M}+\mathrm{H})^{+}$.

2-(Ethylthio)-6-(trifluoromethyl)benzo[d]thiazole (Table 1, Entry 5, 3e): ${ }^{1} \mathrm{H}$ NMR $\left(300 \mathrm{MHz}, \mathrm{CDCl}_{3}, \delta, \mathrm{ppm}\right): 8.03$ (s, $1 \mathrm{H}, \mathrm{Ar}-$ $\mathrm{H}), 7.92(\mathrm{~d}, 1 \mathrm{H}, J=8.3 \mathrm{~Hz}, \mathrm{Ar}-\mathrm{H}), 7.64(\mathrm{~d}, 1 \mathrm{H}, J=9.0 \mathrm{~Hz}, \mathrm{Ar}-\mathrm{H})$, 3.58-3.22 (m, $\left.2 \mathrm{H}, \mathrm{CH}_{2}\right), 1.60-1.39\left(\mathrm{t}, 3 \mathrm{H}, \mathrm{CH}_{3}\right) .{ }^{13} \mathrm{C}$ NMR $(75$ $\left.\mathrm{MHz}, \mathrm{CDCl}_{3}, \delta, \mathrm{ppm}\right): 170.8$ (Ar-C), $155.2(\mathrm{Ar}-\mathrm{C}), 135.1$ (Ar-C), 125.8 (Ar-C), 122.9 (Ar-C), 121.3 (Ar-C), 118.4 (Ar-C), 27.8, AliC, 14.3, Ali-C.

ESI-MS $(m / z): 264(\mathrm{M}+\mathrm{H})^{+}$.

2-(Pentylthio)-6-(trifluoromethyl)benzo[d]thiazole (Table 1, Entry 6, 3f): ${ }^{1} \mathrm{H}$ NMR $\left(300 \mathrm{MHz}, \mathrm{CDCl}_{3}, \delta, \mathrm{ppm}\right):$ 8.09-8.01 (s, $1 \mathrm{H}, \mathrm{Ar}-\mathrm{H}), 8.95-8.90$ (d, $1 \mathrm{H}, \mathrm{Ar}-\mathrm{H}), 7.69-7.55$ (d, $1 \mathrm{H}, \mathrm{Ar}-\mathrm{H}), 3.45-$ $3.28\left(\mathrm{t}, 2 \mathrm{H}, \mathrm{CH}_{2}\right), 1.87-1.70\left(\mathrm{~m}, 6 \mathrm{H}, \mathrm{CH}_{2}\right), 0.93-0.83\left(\mathrm{~s}, 3 \mathrm{H}, \mathrm{CH}_{3}\right)$. ESI-MS $(m / z): 306(M+H)^{+}$.

2-(Heptylthio)-6-(trifluoromethyl)benzo[d]thiazole (Table 1, Entry 7, 3g): ${ }^{1} \mathrm{H}$ NMR $\left(300 \mathrm{MHz}, \mathrm{CDCl}_{3}, \delta, \mathrm{ppm}\right): 8.03$ (s, $1 \mathrm{H}, \mathrm{Ar}-$ H), $7.92(\mathrm{~d}, 1 \mathrm{H}, J=8.4 \mathrm{~Hz}, \mathrm{Ar}-\mathrm{H}), 7.77-7.69$ (d, $1 \mathrm{H}, \mathrm{Ar}-\mathrm{H}), 3.48-$ $3.28\left(\mathrm{t}, 2 \mathrm{H}, \mathrm{CH}_{2}\right), 1.99-1.75\left(\mathrm{~m}, 2 \mathrm{H}, \mathrm{CH}_{2}\right), 1.40-1.20\left(\mathrm{~m}, 8 \mathrm{H}, \mathrm{CH}_{2}\right)$, 0.97-0.78 (m, 3H, $\left.\mathrm{CH}_{3}\right) .{ }^{13} \mathrm{C}$ NMR (75 MHz, $\left.\mathrm{CDCl}_{3}, \delta, \mathrm{ppm}\right): 171.2$ (Ar-C), 155.3 (Ar-C), 135.2 (Ar-C), 123.1 (Ar-C), 121.4 (Ar-C), 118.4 (Ar-C), 33.6, Ali-C, 31.6, Ali-C, 29.0, Ali-C, 28.7, Ali-C, 22.5, Ali-C, 14.0, Ali-C. ESI-MS (m/z): $334(\mathrm{M}+\mathrm{H})^{+}$.
2-(Thiophen-2-ylthio)-6-(trifluoromethyl)benzo[d]thiazole (Table 1, Entry 8, 3h): ${ }^{1} \mathrm{H}$ NMR $\left(300 \mathrm{MHz}, \mathrm{CDCl}_{3}, \delta\right.$, ppm): 8.15$8.00(\mathrm{~m}, 1 \mathrm{H}, \mathrm{Ar}-\mathrm{H}), 7.97-7.90(\mathrm{~m}, 1 \mathrm{H}, \mathrm{Ar}-\mathrm{H}), 7.73-7.69(\mathrm{~m}, 1 \mathrm{H}$, $\mathrm{Ar}-\mathrm{H}), 7.65(\mathrm{~d}, 1 \mathrm{H}, J=8.3 \mathrm{~Hz}, \mathrm{Ar}-\mathrm{H}), 7.35-7.30(\mathrm{~m}, 1 \mathrm{H}, \mathrm{Ar}-\mathrm{H})$, 7.23-7.18 (m, 1H, Ar-H). ESI-MS ( $m / z): 317(\mathrm{M}+\mathrm{H})^{+}$.

2-(Pyridin-2-ylthio)-6-(trifluoromethyl)benzo[d] thiazole (Table 1, Entry 9, 3i): ${ }^{1} \mathrm{H}$ NMR (300 MHz, $\left.\mathrm{CDCl}_{3}, \delta, \mathrm{ppm}\right): 8.42-$ $8.35(\mathrm{~m}, 2 \mathrm{H}, \mathrm{Ar}-\mathrm{H}), 7.77-7.70$ (d, 2H, Ar-H), 7.37-7.32 (m, 1H, Ar-H), 7.31-7.28 (m, 2H, Ar-H). ESI-MS (m/z): $313(\mathrm{M}+\mathrm{H})^{+}$.

2-((4-Methoxyphenyl)thio)-6-(trifluoromethyl)benzo[d] thiazole (Table 1, Entry 10, 3j): ${ }^{1} \mathrm{H}$ NMR $\left(300 \mathrm{MHz}, \mathrm{CDCl}_{3}, \delta\right.$, ppm): 7.17-7.05 (m, 2H, Ar-H), 6.88-6.70 (m, 4H, Ar-H), 6.66$6.55(\mathrm{~m}, 1 \mathrm{H}, \mathrm{Ar}-\mathrm{H}), 3.66\left(\mathrm{~s}, 3 \mathrm{H}, \mathrm{OCH}_{3}\right)$. ESI-MS $(\mathrm{m} / \mathrm{z}): 342(\mathrm{M}+$ $\mathrm{H})^{+}$.

6-(Trifluoromethyl)benzo[d]thiazole-2-thiol (Intermediate, A1): ${ }^{1} \mathrm{H}$ NMR $\left(300 \mathrm{MHz}, \mathrm{CDCl}_{3}, \delta, \mathrm{ppm}\right): 11.08-10.88(\mathrm{~m}, 1 \mathrm{H}, \mathrm{S}-$ H), 7.77-7.72 (s, 1H, Ar-H), 7.67-7.59 (d, 1H, Ar-H), 7.39-7.32 (d, $1 \mathrm{H}, \mathrm{Ar}-\mathrm{H})$. ESI-MS $(\mathrm{m} / \mathrm{z}): 235(\mathrm{M}+\mathrm{H})^{+}$.

\section{Results and discussion}

Initially, 2-mercapto benzothiazole and iodobenzene were used as model reactants to optimize the reaction conditions such as bases, solvents, and reaction temperature (Table 2). Among, several bases screened, $\mathrm{Cs}_{2} \mathrm{CO}_{3}$ was found to be an excellent base (Table 2, Entry 1). In the presence of bases such as $\mathrm{K}_{2} \mathrm{CO}_{3}, \mathrm{Na}_{2} \mathrm{CO}_{3}$, and $\mathrm{K}_{3} \mathrm{PO}_{4}$, lesser amount of the desired product was obtained (Table 2, Entry 2, 3, 4).

The effect of solvents was also investigated and the highest yield was observed in PEG-400 (polyethylene glycol), while reaction in solvents such as $\mathrm{THF}, \mathrm{CH}_{3} \mathrm{CN}$ and toluene resulted in moderate yields. The experiment confirmed that the reaction 
did not occur in the absence the base (Table 2, Entry 10). Different nano catalysts were screened in this protocol (Table 2, Entries 13-16). When the reaction was conducted at room temperature trace amount of desired product were obtained (Table 2, Entry 5). Ideal temperature for the reaction was found to be $90^{\circ} \mathrm{C}$. The influence of the amount of catalyst on the yield of the product was also evaluated. It was observed that 0.06 mmol of nano $\mathrm{CuFe}_{2} \mathrm{O}_{4}$ was ideal for synthesis of 2-thiosubstituted benzothiazoles under ligand-free conditions.

The reusability of the nano copper ferrite catalyst was examined and the results are summarized in Figure 3.

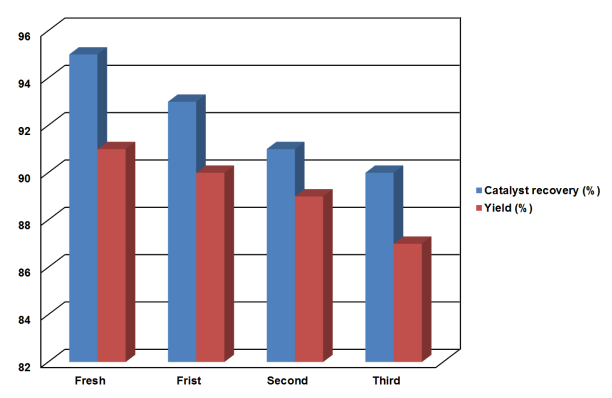

Figure 3. Recycling of $\mathrm{CuFe}_{2} \mathrm{O}_{4}$ nanoparticles (Reaction conditions: A mixture of 2-mercapto benzothiazole $(0.5 \mathrm{mmol})$, iodo benzene $(0.6 \mathrm{mmol}), \mathrm{CuFe}_{2} \mathrm{O}_{4}$ nanoparticles $(0.06 \mathrm{mmol})$, and $\mathrm{Cs}_{2} \mathrm{CO}_{3}(1.3 \mathrm{mmol})$ in PEG-400 $(3 \mathrm{~mL})$ was stirred at $90{ }^{\circ} \mathrm{C}$ for $8 \mathrm{~h}$ ).

After completion of the reaction, $\mathrm{CuFe}_{2} \mathrm{O}_{4}$ nano particles were allowed to be at the bottom of the flask by a neodymium magnet, and the supernatant solution was removed. The separated nano catalyst was washed successively with $10 \mathrm{~mL}$ of water, ethanol, ethyl acetate, dichloromethane, and hexane and then dried. No significant loss of catalyst activity was observed up to four cycles. The native and used $\mathrm{CuFe}_{2} \mathrm{O}_{4}$ nanoparticles were analyzed by powder XRD and SEM analysis. The SEM studies showed that the morphology of $\mathrm{CuFe}_{2} \mathrm{O}_{4}$ was almost similar in both before and after the reaction conditions. The powder XRD spectra (Figure 4 and 5) also confirmed the intactness of the particles after four cycles (supporting. information).

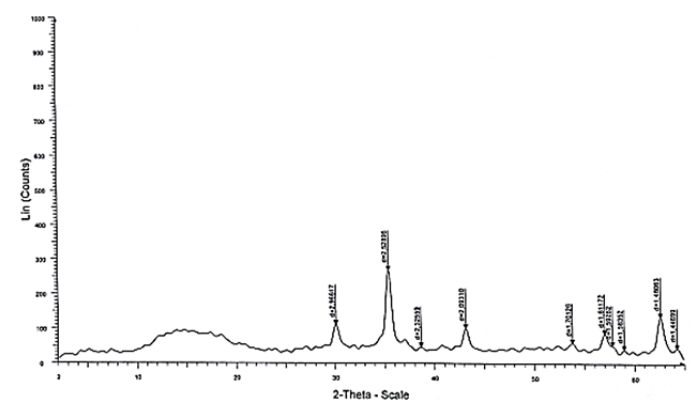

Figure 4. Powder XRD - images of Native $\mathrm{CuFe}_{2} \mathrm{O}_{4}$ nanoparticles.

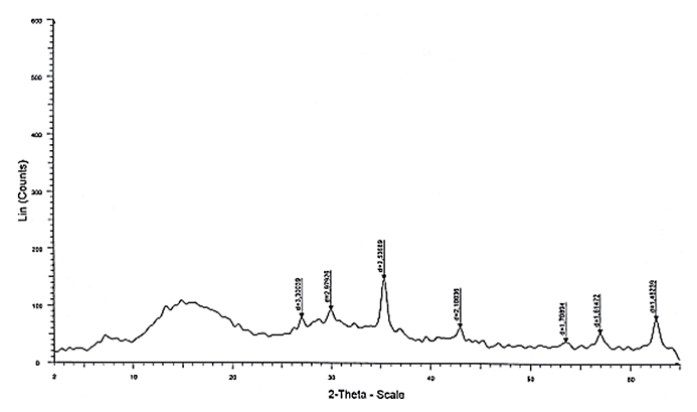

Figure 5. Powder XRD - images of $\mathrm{CuFe}_{2} \mathrm{O}_{4}$ nanoparticles after four cycles.

\section{Conclusion}

A simple and green protocol was developed for the synthesis of 2-thio-substituted benzothiazoles using recyclable $\mathrm{CuFe}_{2} \mathrm{O}_{4}$ nanoparticles under ligand free conditions in an ecofriendly PEG-400 solvent.

\section{Acknowledgement}

We thank Council of Scientific and Industrial Research, New Delhi, India, for fellowship to, Gaddam Satish, Kasireddy Harsha Vardhan Reddy, Jilla Shankar and University Grants Commission for fellowship to Katla Ramesh.

\section{References}

[1]. Koci, J.; Klimesova, V.; Waisser, K.; Kaustova, J.; Dahse, H. M.; Moellmann, U. Bioorg. Med. Chem. Lett. 2002, 12, 3275-3278.

[2]. Zhang L.; Fan, J.; Vu, K.; Hong, K.; Le Brazidec, J. Y.; Shi, J.; Biamonte, M.; Busch, D. J.; Lough, R. E.; Grecko, R.; Ran, Y.; Sensintaffar, J. L.; Kamal, A.; Lundgren, K.; Burrows, F. J.; Mansfield, R.; Timony, G. A.; Ulm, E. H.; Kasibhatla, S. R.; Boehm, M. F. J. Med. Chem. 2006, 49, 5352 5355.

[3]. Dumas, J.; Brittelli, D.; Chen, J.; Dixon, B.; Hatoum-Mokdad, H.; Konig G.; Sibley, R.; Witowsky, J.; Wong, S. Bioorg. Med. Chem. Lett. 1999, 9, 2531-2535.

[4]. Paramashivappa, P.; Kumar, P.; Rao, S. P. V.; Rao, A. S. Bioorg. Med. Chem. Lett. 2003, 13, 657-659.

[5]. Naya, K.; Kobayashi, K.; Ishikawa, M.; Ohwaki, K.; Saeki, T.; Noguchi, K.; Ohtake, N. Chem. Pharm. Bull. 2003, 51(6), 697-701.

[6]. Pejin, C.; Iodice, G.; Tommonaro, S.; Rosa, D. J. Nat. Prod. 2008, 71, 1850-1854.

[7]. Sorg, A.; Bruckner, R. Synlett. 2005, 2, 289-293.

[8]. Liebeskind, L. S. Org. Lett. 2002, 4, 979-982.

[9]. Dunn, P. J. Chem. Soc. Rev. 2012, 41, 1452-1456.

[10]. Toda, F.; Tanaka, K. Chem. Rev. 2000, 100, 1025-1029.

[11]. Zhang, Z. H.; Yin, L.; Wang, Y. M.; Liu, J. Y.; Li, Y. Green Chem. 2004, 6, 563-565.

[12]. Chen, J.; Spear, S. K.; Huddleston, J. G.; Rogers, R. D. Green Chem. 2005 7, 64-67.

[13]. Ferravoschi, P.; Fiecchi, A.; Grisenti, P.; Santaniello, E.; Trave, S. Synth Commun. 1987, 17, 1569-1573.

[14]. Blanton, J. R. Synth. Commun. 1997, 27, 2093-2095.

[15]. Chandrasekhar, S.; Narsihmulu, Ch.; Sultana, S. S.; Reddy, N. R. K. Org. Lett. 2002, 4, 4399-4383.

[16]. Chandrasekhar, S.; Narsihmulu, C.; Sultana, S. S.; Reddy, N. R. K. Chem. Commun. 2003, 1716-1717.

[17]. Namboodiri, V. V.; Varama, R. S. Green Chem. 2001, 3, 146-149.

[18]. Haimov, A.; Neumann, R. Chem. Commun. 2002, 876-879.

[19]. Chandrasekhar, S.; Narsihmulu, C.; Chandrasekhar, G.; Shyamsundar, T. Tetrahedron Lett. 2004, 45, 2421-3425.

[20]. Liu, Shi.; Liu, X.; Zhang, H.; Jiang, Y.; Ma, D. J. Org. Chem. 2011, 76, 420424.

[21]. Wei-Yi, W.; Jui-Chan, W.; Fu-Yu, T. Green Chem. 2009, 11, 326-329.

[22]. Swapna, K.; Murthy, S. N.; Jyothi, M. T.; Nageswar, Y. V. D. Org. Bio. Chem. 2011, 9, 5978-5982.

[23]. Swapna, K.; Murthy, S. N.; Jyothi, M. T.; Nageswar, Y. V. D. Org. Bio Chem. 2011, 9, 5989-5993.

[24]. Reddy, K. H. V.; Reddy, V. P.; Shanka, J.; Madhav, B.; Kumar, B. S. P. A.; Nageswar, Y. V. D. Tetrahedron Lett. 2011, 52, 2679-2682.

[25]. Reddy, K. H. V.; Reddy, V. P.; Shanka, J.; Nageswar, Y. V. D. Synlett 2011, 9, 1268-1271.

[26]. Reddy, K. H. V.; Satish, G.; Ramesh, K.; Karnakar K.; Nageswar, Y. V. D. Chem. Lett. 2012, 41, 585-587.

[27]. Kumar, B. S. P. A.; Reddy, K. H. V.; Madhav, B.; Ramesh K.; Nageswar, Y. V. D. Tetrahedron Lett. 2012, 53, 4595-4598.

[28]. Reddy, K. H. V.; Satish, G.; Ramesh, K.; Karnakar K.; Nageswar, Y. V. D. Tetrahedron Lett. 2012, 53, 3061-3065.

[29]. Reddy, K. H. V.; Satish, G.; Prakash Reddy, V.; Anil, B. S. P.; Nageswar, Y. V. D. RSC Advance 2012, 2, 11084

[30]. Satish, G.; Reddy, K. H. V.; Karnakar, K.; Ramesh, K.; Nageswar, Y. V. D. Tetrahedron Lett. 2012, 53, 2518-2521.

[31]. Wang, F.; Cai, S.; Wang, Z.; Xi, C. Org. Lett. 2011, 13, 3203-3206. 